\title{
Is there a true Model-D critical dynamics?
}

\author{
Parongama Sen \\ Department of Physics, University of Calcutta, 92 A.P. C. Road, Kolkata 700009, India.
}

\author{
Somendra M. Bhattacharjee \\ Institute of Physics, Bhubaneswar 751 005, Indiaf
}

\begin{abstract}
We show that non-locality in the conservation of both the order parameter and a noncritical density (model D dynamics) leads to new fixed points for critical dynamics. Depending upon the parameters characterizing the non-locality in the two fields, we find four regions: (i) model-A like where both the conservations are irrelevant (ii) model B-like with the conservation in the order parameter field relevant and the conservation in the coupling field irrelevant (iii) model $\mathrm{C}$ like where the conservation in the order parameter field is irrelevant but the conservation in the coupling field is relevant, and (iv) model D-like where both the conservations are relevant. While the first three behaviours are already known in dynamical critical phenomena, the last one is a novel phenomena due entirely to the non-locality in the two fields.
\end{abstract}

Based on the long time behaviour, the dynamics of different critical systems are classified [1] in various dynamic universality classes. These classes are characterized by several factors like the number of conserved field, nonvanishing Poisson brackets etc, in addition to the usual quantities necessary for equilibrium. Among the models which have no propagating modes, Model A is a system in which there are no conserved fields, Model B is a system with a conserved order parameter while Model $\mathrm{C}$ is the class of systems with a non-conserved order parameter field coupled to a conservative field. A fourth classification was also made - Model $\mathrm{D}$, in which both the order parameter field and the coupled field are conserved. This model, however, was shown to be equivalent to Model B as the coupling to the field becomes irrelevant in the large length long time scale limit.

The dynamical behaviour in systems with one or more conserved field like Models B, C and D in the above classification is usually studied by considering local conservation. However, conservation is by no means necessarily local and in fact, non-local conservation is a more general condition. A few recent studies on critical or nearcritical dynamics show that non-locality in the conserved field may change the dynamical class, besides the obvious modifications in nonuniversal details. This kind of effect of non-locality in the conservation has been noted in the study of critical dynamics of model $\mathrm{B}$ and $\mathrm{C}$ and in the numerical study of the early time effect of a model believed to belong to the class of model $\mathrm{C}$ and on the zero temperature dynamics (phase-ordering kinetics or Ostwald ripening) [2, 3, 4, 5, 6, 7]. Besides the theoretical interest on its own right, one of the important applications of nonlocal dynamics is in speeding up numerical simulations, prompting implementation of various types of nonlocal moves [8]. Hence the need of a general classification of effects of nonlocality in dynamics.

In a sense, model D is the most generalised class of dynamical critical phenomena with dissipative dynamics. However, with local conservation, it is equivalent to
Model B showing no novel features. Motivated by the results of model C[4], we investigate the problem of nonlocal conservation in Model D in the most general way by keeping both the conservations in the order parameter field and the coupled field non-local. One of our main results is to show the emergence of a true model D behaviour by nonlocal dynamics, unlike the local conservation case.

We introduce non-locality by using a non-local kernel 12, 3, 4] in the chemical potential keeping the continuity equation intact. Using $\phi$ for the order parameter and $m$ for the secondary coupling parameter, the Hamiltonian of model D is given by

$$
\begin{array}{r}
H=\int d^{d} \mathbf{x}\left[\frac{1}{2} r \phi^{2}(\mathbf{x})+\frac{1}{2}(\nabla \phi(\mathbf{x}))^{2}+\tilde{u} \phi^{4}(\mathbf{x})\right. \\
\left.+\gamma \phi^{2}(\mathbf{x}) m(\mathbf{x})+\frac{1}{2} C^{-1} m^{2}(\mathbf{x})\right],
\end{array}
$$

where $\phi^{2}(\mathbf{x})=\sum_{i=1}^{n} \phi_{i}^{2}(\mathbf{x}), \phi^{4}(\mathbf{x})=\left[\sum_{i=1}^{n} \phi_{i}^{2}(\mathbf{x})\right]^{2}, r=$ 0 is the mean field critical point, and $C>0$ ensures noncriticality of $m$. There is a short distance cutoff which in momentum space is an ultraviolet cutoff $\Lambda$. We shall set $\Lambda=1$ in the calculation. The above Hamiltonian, Eq. (11) is the same as in Model C[1], 朋. Making $\gamma=0$ effectively reduces the above model to model A.

The dynamics ${ }^{[\dagger]}$ is given by the following equations:

$$
\begin{aligned}
\left(\frac{1}{\Gamma_{\rho} k^{\rho}}+\frac{1}{\Gamma_{0}}\right) \frac{\partial \phi_{\mathbf{k}}}{\partial t} & =-\frac{\delta H}{\delta \phi_{-\mathbf{k}}}+\eta(\mathbf{k}, t) \\
\left(\frac{1}{\lambda_{\sigma} k^{\sigma}}+\frac{1}{\lambda_{0}}\right) \frac{\partial m_{\mathbf{k}}}{\partial t} & =-\frac{\delta H}{\delta m_{-\mathbf{k}}}+\zeta(\mathbf{k}, t) .
\end{aligned}
$$

[††] Additional torques for $n=3$ (Hejsenberg model) may lead to new dynamic universality classes 96. We do not consider such effects. 
The noises $\eta$ and $\zeta$ obey

$$
\begin{array}{r}
\langle\eta\rangle=0,\langle\zeta\rangle=0,(3 \mathrm{a}) \\
\left\langle\eta(\mathbf{k}, t) \eta\left(\mathbf{k}^{\prime}, t^{\prime}\right)\right\rangle=2\left(\frac{1}{\Gamma_{\rho} k^{\rho}}+\frac{1}{\Gamma_{0}}\right) \delta\left(\mathbf{k}+\mathbf{k}^{\prime}\right) \delta\left(t-t^{\prime}\right),(3 \mathrm{~b}) \\
\left\langle\zeta(\mathbf{k}, t) \zeta\left(\mathbf{k}^{\prime}, t^{\prime}\right)\right\rangle=2\left(\frac{1}{\lambda_{\sigma} k^{\rho}}+\frac{1}{\lambda_{0}}\right) \delta\left(\mathbf{k}+\mathbf{k}^{\prime}\right) \delta\left(t-t^{\prime}\right) .(3 \mathrm{c})
\end{array}
$$

Here, $k=|\mathbf{k}|$, and we have introduced two different parameters $\rho$ and $\sigma$ to denote the non-localities in the order parameter field and the coupling field respectively. The dissipative or the kinetic coefficients $\left(\Gamma_{0}\right.$ and $\left.\lambda_{0}\right)$ are denoted by the subscript 0 while the transport coefficients $\left(\Gamma_{\rho}\right.$ and $\left.\lambda_{\sigma}\right)$ by the corresponding powers of $k$. For the local case, both $\rho$ and $\sigma$ assume a value equal to 2. Results are known for some limiting values of $\rho$ and $\sigma$ : a) $(\rho=0, \gamma=0)$ corresponds to model A [1], b) $(\rho=2, \gamma=0)$ and $(\rho=2, \sigma=2)$ give model B like behaviour [1, c) any value of $\rho>0$ and $\gamma=0$ is a non-local Model B 2 2, 5], and d) $\rho=0$ with any $\sigma>0$ is non-local model C 画.

A Hamiltonian of the type of Eq. 11 occurs near the critical wings of a tricritical system or in critical systems with additional constraints. Model D dynamics are relevant in such cases, for example in three or four component mixtures or in spin- 1 or Potts models 10]. A spin- $\frac{1}{2}$ example, though a bit unrealistic, would be an antiferromagnetic system with conserved magnetisation (as in model C [6, 11]) as well as conserved staggered magnetisation. The Kawasaki dynamics can be used to conserve the magnetisation maintaining at the same time that the exchanges between opposite spins have to be done in such a way that the order parameter is unchanged.

A momentum-shell renormalization group approach is used to study the long distance long time behaviour. In this approach, small scale fluctuations in space (between $\Lambda e^{-\delta l}$ and $\Lambda$ in momentum space) are integrated out, and the cutoff rescaled to $\Lambda$. One then obtains an effective Hamiltonian and effective equations for the dynamics valid for longer scales. The universal features are obtained from the fixed points of the flow equations for the various parameters of the problem. As per the usual RG scheme, any parameter that vanishes (grows) in the long scale limit is called an irrelevant (relevant) variable. A fixed point is then characterized by the set of relevant parameters. In this scheme the four dynamics classes correspond to the relevance and irrelevance of $\lambda_{\sigma}$ and $\Gamma_{\rho}$ as mentioned in the abstract.

The statics of this system is the same as in Model C and the fixed point values of the parameters $r, u$ and $\gamma^{2} C$ are as given in ref [4]. The dynamical equations, on the other hand, take the forms:

$$
\begin{aligned}
\frac{\partial \lambda_{\sigma}^{-1}}{\partial l} & =\left(-z+\sigma+\frac{\alpha}{\nu}\right) \lambda_{\sigma}^{-1} \\
\frac{\partial \lambda_{0}^{-1}}{\partial l} & =\left(-z+\frac{\alpha}{\nu}\right) \lambda_{0}^{-1}+\left(n \gamma^{2} K_{d}\right) Q \\
\frac{\partial \Gamma_{\rho}^{-1}}{\partial l} & =(-z+\rho+2) \Gamma_{\rho}^{-1} \\
\frac{\partial \Gamma_{0}^{-1}}{\partial l} & =(-z+2) \Gamma_{0}^{-1}+\left(4 C \gamma^{2} K_{d}\right) Q \frac{1}{1+\frac{Q}{C P}}
\end{aligned}
$$

where $Q=\Gamma_{\rho}^{-1}+\Gamma_{0}^{-1}, P=\lambda_{\sigma}^{-1}+\lambda_{0}^{-1}$ and $K_{d}=$ $2^{1-d} \pi^{-d / 2} \Gamma(d / 2)$. The parameters $\lambda_{\sigma}, \lambda_{0}, \Gamma_{\rho}, \Gamma_{0}, \gamma$ and $C$ appearing in the above equations are all $l$ dependent. To simplify notation, this $l$-dependence has been suppressed.

From (4b) we get

$$
\frac{\Gamma_{0}}{\lambda_{0}}=\frac{(1+x) n \gamma^{2} K_{d}}{z-\alpha / \nu}
$$

where $x=\frac{\Gamma_{0}}{\Gamma_{\rho}}$ is the dimensionless ratio of the two coefficients for the primary order-parameter (note: $\Lambda=1$ ). This quantity $x$ satisfies

$$
\frac{\partial x}{\partial l}=(\rho-Y) x, \quad \text { where } \quad Y=\frac{2 \alpha}{\nu n} \frac{1}{\mu^{-1}+(x+1)^{-1}} .
$$

Here we have introduced the ratios of the transport and kinetic coefficients of $m$ with the kinetic coefficient of $\phi$, namely,

$$
\mu_{\sigma}=\frac{\Gamma_{0} C}{\lambda_{\sigma}}, \quad \mu_{0}=\frac{\Gamma_{0} C}{\lambda_{0}} \quad \text { and } \mu=\mu_{\sigma}+\mu_{0} .
$$

By using the fixed point value of $C$ and Eq. (5), $\mu_{0}$ can be rewritten as

$$
\mu_{0}=\frac{(x+1) \alpha / \nu}{2(z-\alpha / \nu)} .
$$

The flow equation for $\mu_{\sigma}$ is

$$
\frac{\partial \mu_{\sigma}}{\partial l}=\mu_{\sigma}[\sigma-2+\alpha / \nu-Y]
$$

We consider the different solutions of the equations for $x$ and $\mu_{\sigma}$. Corresponding to the relevance of the conserved quantities, one gets different regions in the $\rho-\sigma$ plane with different dynamic critical behaviour. It should be mentioned here that for $n \geq 4$, the coupling $\gamma$ scales to zero and one effectively gets a non-local Model B. This has already been considered and shown to be model Alike when globally conserved [2]. Our discussions are for the $n<4$ region where the coupling survives.

There are three fixed points of $x: x=0, x=\infty$ and a non-zero finite fixed point value for $x$ from Eq. (6): 
Case 1. $x=0$ in the long length scale limit:

This corresponds to non-local Model C: the conservation of the order parameter is irrelevant. The fixed points and their stabilities of non-local model $\mathrm{C}$, which were obtained in Ref. [- from the solutions of $\mu_{\sigma}$, now depends on the value of $\rho$. In general, $x=0$ will be a stable fixed point as long as $\rho<Y$. We focus on the stability of the various regions for non-zero values of $\rho$.

(a) $\mu_{\sigma}$ has a finite fixed point value and $z=\sigma+\alpha / \nu$. This is a stable solution in the region $(2 / n)-1>p>-1$ (where $p=\frac{\sigma-2}{\alpha / \nu}$ ) for $\rho=0$. For non-zero $\rho$ this remains stable for $\rho<p+1$.

(b) $\mu_{\sigma}=\infty$ : this is valid for $p>(2 / n)-1$. Here $z=2+2 \alpha / n \nu$. This solution $x=0, \mu_{\sigma}=\infty$ is stable as long as $\rho<2 \alpha / n \nu$.

(c) For $\mu_{\sigma}=0$, the stability of $x=0$ is valid only for $p<-1$ and $\rho<O\left(\epsilon^{2}\right)$. Here $z=2+O\left(\epsilon^{2}\right)$ and the behaviour is model A like. Any non-zero value of $\rho$ thus destroys this model A like region. Therefore, a region where both the conservations are irrelevant is restricted strictly to $\rho=0$.

Case 2. A finite non-zero value of $x$ in the long length scale limit: This occurs when $\rho=Y$. In order that $\Gamma_{0}$ reaches a finite fixed point value, we must have $z=2+\rho$. In this case, $\mu_{\sigma}$ again has the following fixed points:

(a) $\mu_{\sigma}=\infty$ for $\sigma-2+\alpha / \nu>\rho$

(b) $\mu_{\sigma}=0$ for $\sigma-2+\alpha / \nu<\rho$

(c) $\mu_{\sigma}$ nonzero finite for $\sigma-2+\alpha / \nu=\rho$

Physically a finite valued fixed point of $x$ means that the conservation in the order parameter $\phi$ is relevant. If at the same time $\mu_{\sigma}$ attains a non-zero fixed point value, the conservation of the coupling field $m$ is also relevant. This occurs if the fixed points (a) and (c) are stable.

For case (a), $x=\frac{\rho-2 \alpha / \nu n}{2 \alpha / \nu n}$ and for non-negative value of $x, \rho>2 \alpha / n \nu$. Hence in between $\rho=2 \alpha / n \nu$ and $\rho=\sigma-2+\alpha / \nu$, a stable region is obtained where both the conservations are relevant.

For case (b), $x+1=\frac{\rho(2 z-\alpha / \nu)}{2 \alpha^{2} / n \nu^{2}}$. This has a finite valued solution for $x$ only for $\rho \sim O\left(\epsilon^{2}\right)$. Hence for $\rho>$ $\sigma-2+\alpha / \nu, x \rightarrow \infty$ is the only solution for $\mu=0$. It is a conventional model B fixed point.

Case (c): Here $z=\sigma+\alpha / \nu$ and in principle along the entire line $\sigma-2+\alpha / \nu=\rho$ a solution exists where both the conservations are relevant. However, there is a restriction on the value of $\sigma$ from the condition of existence of a nonzero finite value of $\mu_{\sigma}$. We find that

$$
\mu_{\sigma}+\mu_{0}=\frac{(\sigma-2+\alpha / \nu)(x+1)}{2-\sigma-\alpha / \nu+\frac{2 \alpha}{n \nu}(x+1)} .
$$

Hence with $\sigma=2+p \alpha / \nu$; we get the condition $(1+$ $p)<2(x+1) / n$. Since $x$ is also non-zero, this implies that this solution with both $x$ and $\mu_{\sigma}$ finite is valid for $p+1=\rho /(\alpha / \nu)$ with at least $p<(2 / n)-1$.

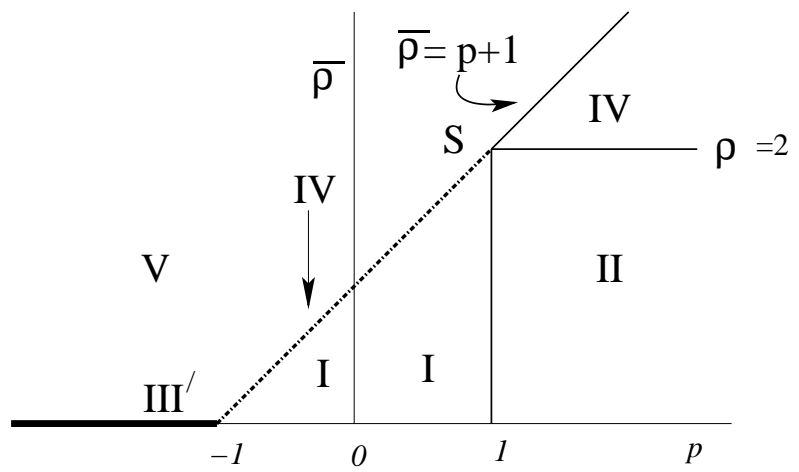

FIG. 1: The different regions in the non-local Model D with $n=1$ is shown in the rescaled $\bar{\rho}-p$ plane where $\bar{\rho}=\rho /(\alpha / \nu)$ and $p=\frac{(\sigma-2)}{\alpha / \nu}$. Regions I and II are model C like with $z=$ $\sigma+\alpha / \nu$ and $z=2+2 \alpha / \nu$ respectively. Region III' is model A like (as in model C of Ref. 4) with $z=2+O\left(\epsilon^{2}\right)$. Reg IV is the "Model D" region, $z=\rho+2$; Reg V is Model B like, $z=\rho+2$. The line $p+1=\rho$ is also model $\mathrm{D}$-like upto point $\mathrm{S}$ (shown by the dashed line). There are no discontinuities of $z$ along any boundary except at the boundary between III' and V.

In the rest of the $\rho-\sigma$ plane, the only solution is $x=\infty$ and $1 / \lambda_{\sigma}=0$ for which only the conservation of the order parameter is relevant. Here $z=\rho+2$.

All the above possibilities of different dynamic behaviours are summarized in Fig. 1 in the $\rho-\sigma$ plane (for $n=1$ ). The possibility of seeing a region where both the conservation conditions are relevant raises new issues like early time effect and boundary or surface effects 12. in the new regime. These remain to be studied.

To summarize, Model D can be viewed as the most general dynamic model with purely dissipative dynamics and we indeed obtain all the four types of behaviours as soon as non-locality in the conservations (as in Eq. (2)) are introduced. Since our general model subsumes the previously studied nonlocal model $\mathrm{C}$ and local model $\mathrm{D}$, it is natural to expect A-like, B-like and C-like regimes. The model A like region, however, turns out to be unstable for any finite value of $\rho$. The model $\mathrm{C}$ like regions are stable for small nonzero values of $\rho$ but ultimately disappear for larger values of $\rho$. The most non-trivial result, for general values of $\rho$ and $\sigma$, is the appearance (Region IV in Fig. 1) of a region with a new dynamical behaviour where both the conservations are relevant. This we call a true model D like region - a consequence of nonlocal conservation laws.

Acknowledgments: PS acknowledges financial support from DST project SP/S2/M-11/99.

* Electronic address:

parongama@vsnl.net, paro@cubmb.ernet.in

Electronic address: somen@iopb.res.in 
[1] P. C. Hohenberg and B. I . Halperin, Rev. Mod. Phys. 49435 (1977).

[2] A. J. Bray, Adv. Phys. 43357 (1994).

[3] A. J. Bray, Phys. Rev. B 41 6724; Phys. Rev. Lett. 62 2841 (1989).

[4] P. Sen, J. Phys. A 321623 (1999).

[5] C. Sire and S. N. Majumdar, Phys. Rev. E 52244 (1995).

[6] B. Zheng and H. J. Lou, Phys. Rev. E 63066130 (2001).

[7] M. Conti, B. Meerson, A. Peleg, P. V. Sasorov, condmat/0109157.

[8] P. Tamayo and W. Klein, Phys. Rev. Letts. 63, 2757 (1989); 66, 2049 (1991). L. L. Mosley, P. W. Gibbs and N. Jan, J. Stat. Phys.67, 813 (1992).A. D. Rutenberg, Phys. Rev. E 54, 972 (1996). C. Moukarzel and N. Parga,
J. Phys. A22, 943 (1989). D. Stauffer, Int. J. Mod. Phys. C8, 1263 (1997).

[9] J. Das and M. Rao, Phys. Rev. E 57, 5069 (1998); 62, 1601 (2000).

[10] See, e.g., I. D. Lawrie and S. Sarbach, in Phase transitions and critical phenomena, Vol. 9 Ed. by C. Domb and J. Lebowitz (Academic, London, 1984).

[11] P. Sen, S. Dasgupta and D. Stauffer, Eur. Phys. J B 1 107 (1998).

[12] See, e.g., S. Mukherji, Europhys. J. B 8, 423 (1999). S. Dietrich and H. W. Diehl, Z. Phys. B 51, 343(1983). K. Oerding and H. K. Jenssen, J. Phys. A26, 3369 (1993); see also 6. 